\title{
A Survey on Social Network Visualization
}

\author{
Jiang $\mathrm{Du}^{1} \&$ Yafei Xian ${ }^{1}$, Jiayu Yang ${ }^{2}$ \\ ${ }^{1}$ College of Computer Science and Technology, Chongqing University of Posts and \\ Telecommunications \\ ${ }^{2}$ Chongqing iSoft Network Security Information Technology Co., Ltd.
}

Keywords: Social network; Visualization schemes; Visualization layout; Visualization applications.

\begin{abstract}
The rapid development of Micro-blog, Renner and other social networks, on the one hand it is difficult to manage these social networks with figures and tables, on the other hand it is difficult to find a wealth of information in social networks. Visualization technology provides an effective way to understand the social network structure and dig information. This article introduces the social network data features, social network visualization solutions, common layout of social network visualization, and social network visualization application in various fields.
\end{abstract}

\section{Introduction}

With the widespread use of mobile Internet, the development of cloud computing technology, social network, e-commerce, LBS services, the size of social network data increases large, jumped from GB to TB grade level, even PB-class [12] that people cannot manage and analyze these social networks by conventional techniques and methods.

The structure of social network is very complex. Only does use spreadsheet or text to express, is it very difficult to understand the social network and the contained information of the social network is not fully reflected. The best easy and intuitive [13] way is visualization [4]. The idea of scientific visualization is proposed by the National Science Foundation in the 1980s, there were producing a large amount of data in scientific computing, it was difficult to know the indicated meaning of these data and the relationship between these data, so they proposed the visualizing ideas of displaying these data to graph. On the one hand the social network visualization can help people recognize the internal structure of the network by the graphical display, on the other hand can explore valuable information hidden inside the social networks [1].

Visualization of social networks involves sociology, graph theory, information visualization, and other fields. This article focuses on the aspect of visualization, the article elaborated on the characteristics of social network data, visualization schemes of different characteristics data, common visual layout of social networks, applications of social network visualization, and finally a summary of the article content.

\section{Characteristics of social network data}

Human social activities generate a lot of social network data, such as Sina publishes tens of thousands of micro-blogs every day. How to analyze the data of these social networks has become a hot research field[5]. Visualization of social network data using mathematics diagram, graph vertices represent people, links show the relationship between people's activities. Because social networks are a kind of complex networks [2], so social network data is in line with the general characteristics of complex networks, mainly in the following four points:

\subsection{Social network data is sparse as a whole, dense local}

In the Social networks and cooperation networks between scientists, the activities between people who mutual understand or cooperate are very frequently. The data is used to represent these activities is very large, contact between these data will be very close, and these social network data presents locally dense features. From the whole region or country to observe, social network and collaborative 
network of scientists is less contact between the various regions, social network data as a whole experience the feature of sparse.

\subsection{Social network data have time characteristic}

Social network activity is often occured with a time, this time records the whole process of activities, studying of social networks often start from this time. For example, when analyzing the micro-blog public opinion, when, who issued, the number of people forwarding, each time of these actions. Then analyzing the information in chronological order, we can have a certain understanding of the propagation of public opinion.

\subsection{Social network data have hierarchical attribute}

The social network of social structure and family relationships have grade level. A hierarchy contains the following sub-level, as well as the second son of the following sub-level hierarchy. For example, a company's organizational structure, generally divided into chariman, middle manager, the general staff of the three levels, these social networks have a property of hierarchical structure.

\subsection{Social network data have network attribute}

The main participants of the Internet network, social networks, collaboration networks, disease transmission networks is human, exchanging activities between people staggered overlap. The links of visual image between nodes mutually cross, intricate, such social network have network properties.

\section{3. social network visualization schemes}

With a variety of features, one visual scheme does clearly not present the characteristics of the various types of social network data. Different characteristics data need different visualization schemes. This section describes some common social network visualization schemes.

\subsection{Node link diagram}

Node link diagram organizes the data into a structure similar to graph, nodes and links represent data items and relations between them, as shown in Figure 1. Node link diagram is used to visualize data with hierarchical attributes, using the form of a tree diagram layout - tree, the data can be visually show hierarchical relationships, the corporate organizational structure shown in Figure 2. It is also commonly used to visualize network data, the social relationships structure shown in Figure 3.

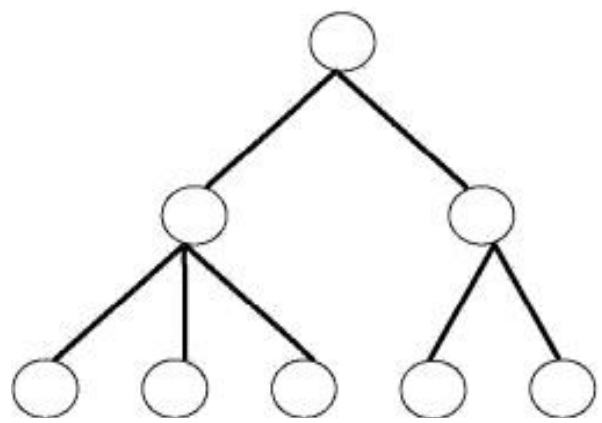

Figure 1. Node link diagram

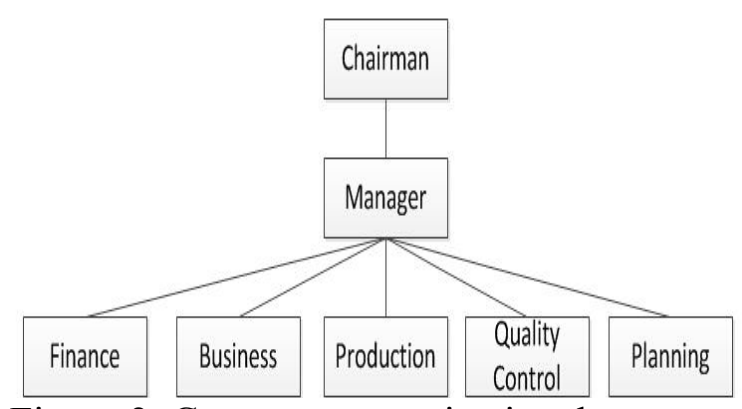

Figure 2. Corporate organizational structure 


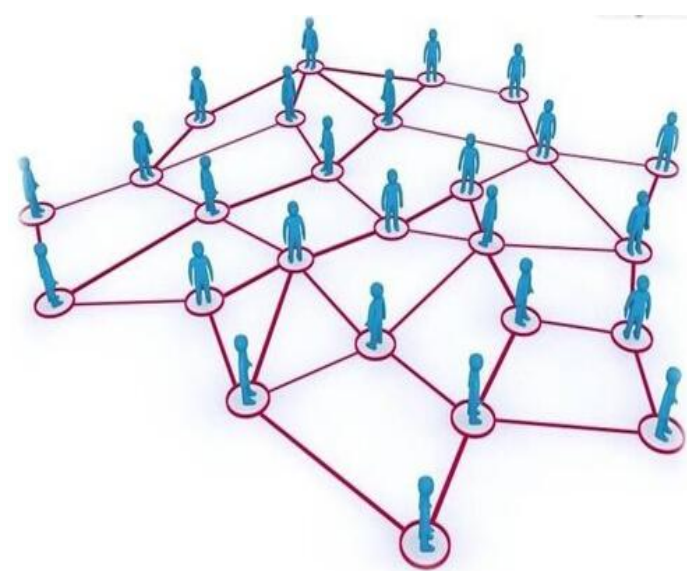

Figure 3. Social relationship graph

\subsection{Matrix}

Node link diagram can show small amount of hierarchy data, network data and there is a lot of space between the branches figure. When the amount of data is large, intertwined branches will become chaotic. Matrix would be a good solution to this problem, all the nodes as a matrix of rows and columns, the matrix value represent the relationship between the nodes, so that the large amount of data, close relationship data can be clearly visualized, as shown in Figure 4.

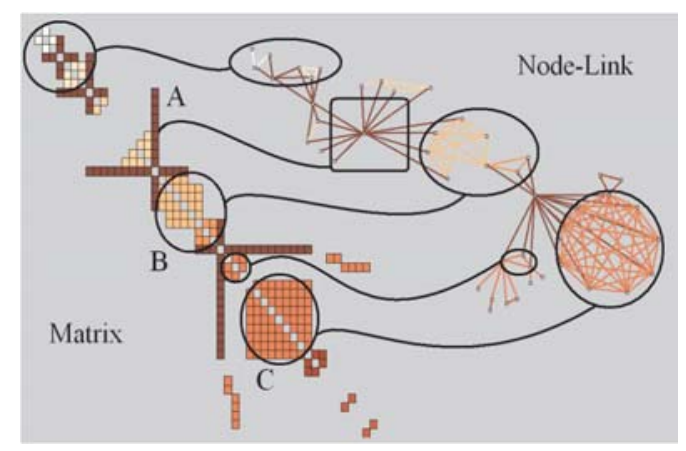

Figure 4. Matrix of interlocking nodes

\subsection{Time line graph}

For the data which has time attribute, information is placed in the horizontal axis coordinate system by chronological order, the time is regard as a clue to study social network, the timeline graph shown in Figure 5. The main problem of time line graph is that the limited length of the time axis is difficult to display all the information when the time is too long .

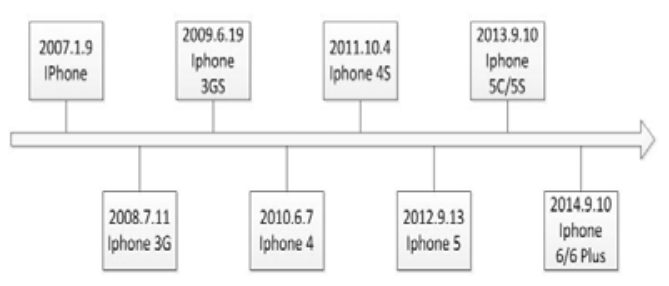

Figure 5. Iphone Publish Time line graph

\subsection{Mixed graph}

The section of papers mentioned that the social network data have overall sparse, dense local features. Theses current visualization scheme cannot clearly visualize these data, researchers have proposed a hybrid visualization scheme, the node link diagram visualizes sparse data and matrix visualizes dense data, mixed graph can clearly visualizes this feature of social network data ,as shown in Figure 6. 


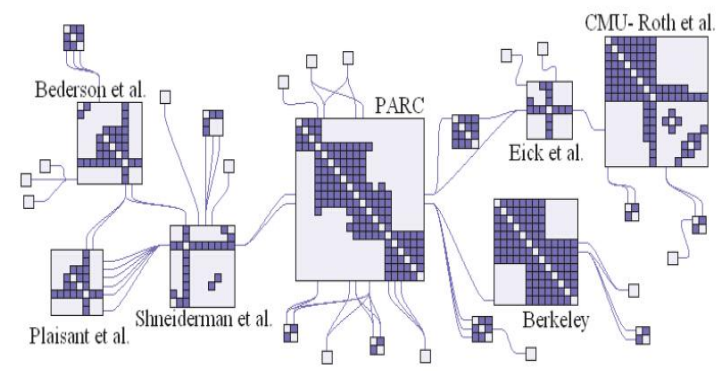

Figure 6. Mixed graph

\section{The layout of social network visualization}

The layout of diagram is the basis of social network visualization layout, the layout of the related research has been very mature, this paper discusses common social network visualization layout focused on the characteristics of social network data.

\subsection{Force directed layout}

The basic idea of force directed layout is to regard graph as a physical system which the vertex act as steel ring, the edge act as spring, when the system is given an initial state, the role of the spring tension will lead to the steel ring moving, it does not stop until the total energy of the system is reduced to the minimum value. Force directed layout is the basis of social network data layout, many layout algorithms are evolved based on it.

\subsection{Hierarchical layout}

Hierarchical layout is mainly for social network data with a hierarchical attribute. Node link diagram with the layout of tree structure, each layer of the tree diagram representing the data of per layer, the hierarchical data is been clearly visualized.

\subsection{Network layout}

Network Layout mainly visualize the social network with network characteristics, the node link diagram was regarded as network to play in a plane, avoiding links cross and nodes overlap.

\section{The application of social network visualization}

Currently, there are many scholars applying social network visualization to the study of social network. Correa et al. use the centrality of node connection in the visualization network to metric key nodes in social networks. $\mathrm{Li}, \mathrm{X}$. et al. who solves the problem that the searching result is too large and complex in the large-scale social network application, recommending the interesting information of user with combining personalization and visualization. Yang, Y.B. et al. who combine social network visual analysis and data mining theory, analyzing the data of 1417 cases of terrorist attacks during the year 1980-2002 with the relevant geographic information, obtained significant results. Huang, M.L. et al. presents a graphical visualization technology of large-scale based on social network visualization joined with hierarchical clustering.

\section{Conclusion}

This paper discusses the characteristics of social network data, the related technology, research and application of social network visualization. Social network visualization, to a certain extent, reduce the difficulty of social network analysis.

\section{References}

[1] Ahmed, A. Dywer, T. Hong, S.H. et al. 2005. Visualization and Analysis of Large and Complex Scale-free Networks. In: EUROGRAPHICS-IEEE VGTC Symposium on Visualization.

[2] Barabasi, A.L. \& Bonabeau, E. 2003. Scale-free networks. Scientific American. 288: 60 69. 
[3] Correa, C.D. Crnovrsanin, T. \& Ma, K.L. 2012. Visual Reasoning about Social Networks Using Centrality Sensitivity. IEEE Transactions on Visualization and Computer Graphics. 18(1): 106-120.

[4] Defanti, T.A. Brown, M.D. \& McCormick, B.H. 1989. Visualization: Expanding scientific and engineering research opportunities. Computer. 22(8): 12-16.

[5] Ding, Z.Y. \& Jia Y. 2014. Survey of Data Mining for Microblogs. Journal of Computer Research and Development. 51(4): 691-706.

[6] Eades, P. A heuristic for graph drawing. Congresses Nutnerant-iunt, 1984, 42: 149 160.

[7] Henry, N. Fekete, J.D. 2006. Matrix Explorer: A dual-representation system to explore social networks. IEEE Trans. on Visualization and Computer Graphics. 12(5):677-684.

[8] Henry, N. Fekete, J.D. McGuffin, M.J. 2007. Node Trix: A hybrid visualization of social networks. IEEE Trans. on Visualization and Computer Graphics. 13(6):1302-1309.

[9] Herman, I. Melancon, G. Marshall, M.S. 2000. Graph visualization and navigation in information visualization: A survey. IEEE Trans. On Visualization and Computer Graphics. 6(1):24-43.

[10]Huang, M.L. \& Nguyen, Q.V. 2008. Large Graph Visualization by Hierarchical Clustering. Journal of Software. 19(8): 1933-1946.

[11]Li, X. \& Cao, L. et al. 2014. Visualization Method of Data Personalized Recommendation in Social Network. Computer Engineering. 40(3): 46-50.

[12]Meng, X.F. \& Ci, X. 2013. Big Data Management: Concepts, Techniques and Challenges. Journal of Computer Research and Development. 50(1):146-169.

[13]Ren, Y.G. \& Yu, G. 2004. Research and Development of the Data Visualization Techniques. Computer Science. 31(12):92-96.

[14] Robertson, G. G. Mack inlay, J.D. Caed, S.K. Cone trees: Animated 3D visualizations of hierarchical information [A]. Proceedings of the SIGCHI Conference on Human Factors in Computing Systems[C]. New York: ACM, 1991.189-194.

[15] Wang, Z.J. \& Huang, W.L. 2013. Research on the Application of Big Data Technology in Query System of Flow Detail Records for Mobile Users. Information and communication technology.

[16] Wu, P. \& Li, S.K. et al. 2011. Layout of Algorithm Suitable for Structural Analysis and Visualization of Social Network. Journal of Software. 22(10): 2467-2475.

[17] Yang, Y.B. \& Liu B. et al. 2014. Review of information Visualization. Journal of Hebei University of Science and Technology. 35(1):91-102.

[18] Yang, Y.B. \& Li, N. et al. 2008. Networked Data Mining Based on Social Network Visualizations. Journal of software. 19(8): 1980-1994.

[19]Zhang, X. \& Yuan, X.R. 2012. Tree map Visualization. Journal of Computer-Aided Design \& Computer Graphics. 24(9): 1113-1124. 